\title{
Analysis of the local structure of phosphorus-substituted LAMOX oxide ion conductors
}

\author{
Bholanath Pahari, Noureddine Mhadhbi, Gwenaël Corbel, Philippe Lacorre and Jens Dittmer*
}

\begin{abstract}
The effect of partial substitution of molybdenum by phosphorus on the global and local structural arrangement of the fast oxide-ion conductor $\mathrm{La}_{2} \mathrm{Mo}_{2} \mathrm{O}_{9}$ (LAMOX) has been studied by X-ray powder diffraction as well as ${ }^{139} \mathrm{La}$ and ${ }^{31} \mathrm{P}$ solid state NMR. The diffraction patterns show that $\mathrm{La}_{2} \mathrm{Mo}_{2-y} \mathrm{P}_{y} \mathrm{O}_{9-y / 2}$ forms a solid solution at low phosphorus concentrations, and that there is a structural phase transition upon increasing phosphorus concentration. This phase transition is also reflected in ${ }^{139} \mathrm{La}$ and ${ }^{31} \mathrm{P}$ NMR spectra. The possibility to excite ${ }^{31} \mathrm{P}$ multiple-quantum coherences of one of the ${ }^{31} \mathrm{P} N \mathrm{NR}$ signals gives evidence of an accumulation of phosphorus atoms on neighbouring Mo-type sites already before formation of three-dimensional precipitates. On the basis of our X-ray and NMR results we propose a possible structural arrangement of the compound $\mathrm{La}_{2} \mathrm{Mo}_{2-y} \mathrm{P}_{y} \mathrm{O}_{9-y / 2}$ that explains the experimental observations by crystal twinning.
\end{abstract}

\section{Introduction}

In recent years solid oxide ion conductors have gained considerable attention due to their technological applications in the fields of solid oxide fuel cells and other electrochemical devices. The finding of materials with high ionic conductivity at lower operating temperatures has become a great challenge to the materials science community. A step forward in this direction was made by the discovery of a new family of oxide ion conductors, the so-called LAMOX family, derived from lanthanum molybdate $\mathrm{La}_{2} \mathrm{Mo}_{2} \mathrm{O}_{9}{ }^{1,2}$ As a matter of fact this latter compound exhibits, above $600{ }^{\circ} \mathrm{C}$, a higher ionic conductivity than YSZ, the reference electrolyte of solid oxide fuel cells. This would allow a lowering of the cell operating temperature by about $150{ }^{\circ} \mathrm{C}$. $\mathrm{La}_{2} \mathrm{Mo}_{2} \mathrm{O}_{9}$ shows a first order structural transition from a monoclinic $(\alpha)$ phase to a cubic $(\beta)$ phase at around $580{ }^{\circ} \mathrm{C}$, which is accompanied by a rise in the conductivity by almost two orders of magnitude. ${ }^{1}$ The structures of the less-conducting monoclinic $(\alpha)$ and highly conducting cubic $(\beta)$ phases have been investigated by several groups. ${ }^{2-4}$ In the $\alpha$ phase there are 48 different Mo sites which can be grouped into three subgroups depending on Mo coordination numbers $(4,5$ or 6$) .{ }^{4}$ In the $\beta$ phase only one Mo site has been observed. The origin of the higher conductivity in the cubic phase has been ascribed to the presence of intrinsic oxygen vacancies in the compound. ${ }^{5}$ The conduction mechanism involves the migration of the oxygen ions through these vacancies. The structural phase transition from $\alpha$ to $\beta$ phase is also understood as a transition from a static to a dynamic distribution of the oxygen vacancies. $4,6,7$

LUNAM Université, Université du Maine, UMR CNRS 6283, Institut des Molécules et Matériaux du Mans (IMMM), Olivier Messiaen, F-72085 Le Mans Cedex, France
Present interest has shifted to the stabilization of the highly conducting $\beta$-phase. Substitution at the cation and/or oxygen sites has proved to be a convenient method to induce it. Numerous substituted systems such as $\left(\mathrm{La}_{2-\mathrm{r}} \mathrm{A}^{n+}{ }_{r}\right) \mathrm{Mo}_{2} \mathrm{O}_{9-(3-n) \times / 2}\left(\mathrm{~A}^{+}\right.$ $=\mathrm{Na},{ }^{8} \mathrm{~K},{ }^{8-10} \mathrm{Rb}^{8} \mathrm{~A}^{2+}=\mathrm{Ca}, \mathrm{Sr}, \mathrm{Ba} ;{ }^{10} \mathrm{~A}^{3+}=\mathrm{Bi},{ }^{11} \mathrm{Pr},{ }^{12} \mathrm{Nd},{ }^{13,14}$ $\left.\mathrm{Eu}^{15}{ }^{15} \mathrm{Gd},{ }^{13} \mathrm{Y}^{13}\right), \mathrm{La}_{2} \mathrm{Mo}_{2-y} \mathrm{~B}^{n+}{ }_{\nu} \mathrm{O}_{9-(6-n) y / 2}\left(\mathrm{~B}^{5+}=\mathrm{V},{ }^{16} \mathrm{Nb},{ }^{17,18}\right.$ $\mathrm{Ta} ;{ }^{18} \mathrm{~B}^{6+}=\mathrm{Cr},{ }^{19} \mathrm{~W}^{19,20}$ and $\mathrm{La}_{2} \mathrm{Mo}_{2} \mathrm{O}_{(9-0.5 y)} \mathrm{F}_{y}{ }^{21}$ have been prepared. In most cases, above a certain substitution rate the high temperature cubic $\beta-\mathrm{La}_{2} \mathrm{Mo}_{2} \mathrm{O}_{9}$ structure is stabilized down to room temperature. Even if this complete stabilization of the $\beta$ phase in the whole thermal range suppresses the conductivity jump at around $580^{\circ} \mathrm{C}$, the conductivity of the substituted/doped compounds above this temperature remains of the same order as that of the parent compound $\beta-\mathrm{La}_{2} \mathrm{Mo}_{2} \mathrm{O}_{9}$. Crystallographically speaking, structural characterization by diffraction techniques, either on powders or on single crystals, only gives a macroscopic averaging of the atomic arrangement. It is important to gain a better insight into how a substitution affects the local structure and the resulting properties of the material. NMR spectroscopy is a good alternative for such a purpose, but its use is limited to those elements with appropriate nuclear spin, gyromagnetic ratio and natural abundance. Phosphorus is a particularly well suited element, since it occurs $100 \%$ as the isotope ${ }^{31} \mathrm{P}$, which has nuclear spin $\frac{1}{2}$ and a relatively high gyromagnetic ratio. It is therefore straightforward to study the local environment of this substituent by ${ }^{31} \mathrm{P}$ NMR. The substitution of phosphorus for molybdenum has up to now never been studied in the LAMOX oxide ion conductors. The first part of this work is devoted to the determination of the existence and extension of the $\mathrm{La}_{2} \mathrm{Mo}_{2-y}$ $\mathrm{P}_{y} \mathrm{O}_{9-y / 2}$ solid solution. Then the study focuses on the characterization, by ${ }^{31} \mathrm{P} \mathrm{NMR}$, of the local environment of phosphorus at different substitution rates in the solid solution. It is complemented by NMR of the spin-7/2 nucleus ${ }^{139} \mathrm{La}$, a nucleus that gives 
extremely broad signals due to quadrupolar coupling. This coupling reflects the electric field gradient (EFG) around the lanthanum cation, a property that is sensitive to the local ground state electronic environment.

\section{Experimental}

\section{Synthesis}

Compositions of the series $\mathrm{La}_{2} \mathrm{Mo}_{2-y} \mathrm{P}_{y} \mathrm{O}_{9-y / 2}$ were prepared by a conventional solid state reaction method from a stoichiometric mixture of $\mathrm{La}_{2} \mathrm{O}_{3},\left(\mathrm{NH}_{4}\right)_{2} \mathrm{HPO}_{4}$ and $\mathrm{MoO}_{3}$ powders. Prior to use, $\mathrm{La}_{2} \mathrm{O}_{3}$ was calcined in air for $12 \mathrm{~h}$ at $1000{ }^{\circ} \mathrm{C}$ in order to remove adsorbed water and carbon dioxide. The well mixed powders were calcined in an alumina crucible at $500{ }^{\circ} \mathrm{C}$ for $12 \mathrm{~h}$. The desired high-purity samples have been obtained after several intermediate grindings and firings in the temperature range $900-1175{ }^{\circ} \mathrm{C}$ (heating and cooling rates of $5{ }^{\circ} \mathrm{C} \mathrm{min}^{-1}$ ).

For the purpose of comparison, the monazite $\mathrm{LaPO}_{4}$ was prepared by heating a stoichiometric mixture of $\mathrm{La}_{2} \mathrm{O}_{3}$ and $\left(\mathrm{NH}_{4}\right)$ $\mathrm{H}_{2} \mathrm{PO}_{4}$ at $500{ }^{\circ} \mathrm{C}$ for 12 hours (heating and cooling rates of $2{ }^{\circ} \mathrm{C}$ $\mathrm{min}^{-1}$ ) and then at $1300{ }^{\circ} \mathrm{C}$ for 12 hours (heating and cooling rates of $5^{\circ} \mathrm{C} \mathrm{min}^{-1}$ ).

\section{X-ray powder diffraction}

Phase purity and identification as well as crystal cell parameters were determined by X-ray powder diffraction at room temperature on a $\theta / \theta$ Bragg-Brentano Philips X'pert MPD PRO diffractometer $\left(\mathrm{Cu} \mathrm{K}_{1+2}\right.$ radiations) equipped with the $\mathrm{X}$ 'celerator detector. For cell parameter refinements, the diffractograms were collected over $300 \mathrm{~min}$ in the scattering angle range [9-130 ${ }^{\circ}$, with a $0.0084^{\circ}$ step, and the program FullProf ${ }^{22}$ was used in the Le Bail's fit mode. ${ }^{23}$ To detect any trace of $\mathrm{LaPO}_{4}$ as impurity, scans were collected during $150 \mathrm{~min}$ in the scattering angle range $\left[27.2-29.2^{\circ}\right]$, with a $0.0084^{\circ}$ step. The integrated intensity of the diffraction peak at $28.64^{\circ}$ was determined using the fit profile option of the software X'Pert HighScore Plus, version 2.0a (PANanalytical B.V., Almelo, Netherlands).

\section{Solid-state NMR}

$4 \mathrm{~mm}$ magic angle spinning (MAS) NMR rotors were filled with equal amounts of $\mathrm{La}_{2} \mathrm{Mo}_{2-y} \mathrm{P}_{y} \mathrm{O}_{9-y / 2}$ for a series of samples with different concentrations $y / 2$ of $\mathrm{P} .{ }^{31} \mathrm{P}$ and ${ }^{139} \mathrm{La}$ NMR experiments were performed on a Bruker Avance III 300 spectrometer operating at a magnetic field of 7.04 T. ${ }^{139}$ La NMR spectra were recorded under static conditions by sweeping the carrier stepwise over the spectral range and acquiring a signal with 1024 scans at each carrier position. For each scan, 10 echoes were generated by multiple refocusing in the QCPMG fashion in intervals of $150 \mu \mathrm{s}^{24}$ The rf strength of the pulses was $93 \mathrm{kHz}\left(p_{90}=\right.$ $2.7 \mu \mathrm{s})$, the recycle delay was $0.2 \mathrm{~s}$. A $50 \mathrm{mM}$ aqueous solution of $\mathrm{LaCl}_{3}$ was used as frequency reference. The echoes were coadded, a Gaussian window of $200 \mathrm{kHz}$ was applied, and the time signal was Fourier transformed. For each partial experiment, the intensity was determined as the $y$-value at the respective carrier frequency. The final spectra consist of the ensemble of these intensities as a function of the carrier frequency. The spectra assembled this way were analyzed by means of the software $d m f i t^{25}$ and a self-written analysis tool. The effect of a Czjzek distribution ${ }^{26}$ was simulated by the "Czjzek simple" model ${ }^{27}$ of $d m f i t$, in which the distributions of chemical shift and quadrupolar coupling are not coupled. For reasons of computing time the chemical shift distribution was adjusted after fitting. All ${ }^{31} \mathrm{P}$ NMR experiments were performed under MAS at either 3, 5 or $7.5 \mathrm{kHz}$ using a $4 \mathrm{~mm}$ dual band probehead. ${ }^{31} \mathrm{P}$ MAS NMR spectra acquired with a spinning rate of $30 \mathrm{kHz}$ did not show a better resolution than those acquired with $7.5 \mathrm{kHz}$ (indicating that the line width is determined by site inhomogeneity rather than chemical shift anisotropy). ${ }^{31} \mathrm{P}$ chemical shifts are referenced to $85 \mathrm{~mol} \% \mathrm{H}_{3} \mathrm{PO}_{4}$ in water. For quantitative 1D spectra 128 scans were acquired with a relaxation delay of $10 \mathrm{~min}$. The set of spectra was deconvoluted in a conjoined fashion by a selfwritten Matlab script. A DQ-SQ experiment was applied to the composition $y=0.04$ with a POST-C $7^{28}$ recoupling time of $1.14 \mathrm{~ms}$ for each direction, 64 increments with 8 scans each, and a relaxation delay of $2 \mathrm{~min}$. For a quantitative analysis, they were applied for a series of samples with 16 increments and between 16 and 176 repetitions. A POST-C7 DQ-SQ experiment with $2.85 \mathrm{~ms}$ recoupling in each direction was combined with a anteceding saturation recovery unit in order to measure longitudinal relaxation times of a DQ-filtered signal. For the sample $y=0.04$, the experiment was done in the 2D fashion with between 16 and 64 repetitions. For spin-counting experiments, ${ }^{29}$ multiplequantum coherences were excited during a mixing period of $2.0 \mathrm{~ms}$ by the application of BABA recoupling ${ }^{30}$ and subsequently reconverted to longitudinal magnetization. The MAS frequency was $3 \mathrm{kHz}$. In order to separate the multiple-quantum coherences, we have used the phase-incrementation scheme of Shykind et al. $^{31}$ with 64 increments. Overall, 200 repetitions have been accumulated for $y=0.04$ and 32 for $\mathrm{LaPO}_{4}$; the interscan delay was $40 \mathrm{~s}$. All NMR experiments have in addition been applied to $\mathrm{LaPO}_{4}$ for sake of comparison.

\section{Results}

\section{Existence and extension of solid solution, phase transition}

Several compounds of the solid solution $\mathrm{La}_{2} \mathrm{Mo}_{2-y} \mathrm{P}_{y} \mathrm{O}_{9-y / 2}$ with compositions $y=0.01,0.02,0.03,0.04,0.05,0.06,0.07,0.08$ and 0.10 were prepared according to the procedure described in the Experimental section, and analyzed by X-ray powder diffraction (300 $\mathrm{min},\left[9-130^{\circ}\right.$ ] range, $0.0084^{\circ}$ step). All compounds up to $y=0.05$ appear to be pure in these patterns, thus attesting the existence of a solid solution. Samples with compositions $y=$ 0.06 to 0.10 show the presence of $\mathrm{LaPO}_{4}$ as impurity phase.

As the phosphorus content $y$ increases, the diffraction patterns reveal a phase transition from a monoclinic $\alpha$ type $(y=0.01$ and $0.02)$ to a cubic $\beta$ type $(y=0.03,0.04$ and 0.05$)$ LAMOX phase (Fig. $1 \mathrm{~A}, 47.5^{\circ}$ ). This is interesting as there is an analogy to the transition to the high temperature, high conductivity cubic $\beta$ phase of the parent compound $\mathrm{La}_{2} \mathrm{Mo}_{2} \mathrm{O}_{9}$.

As a further test of the solid solution domain, the evolution of the crystal cell volume as a function of the $\mathrm{P}$ content is plotted in Fig. 1B. Since phosphorus has a smaller radius than molybdenum $(0.17 \AA$ and $0.41 \AA$, respectively, for $\mathrm{CN}=4),{ }^{32} \mathrm{a}$ decrease of the cell volume was expected up to $y=0.05$; then, in 

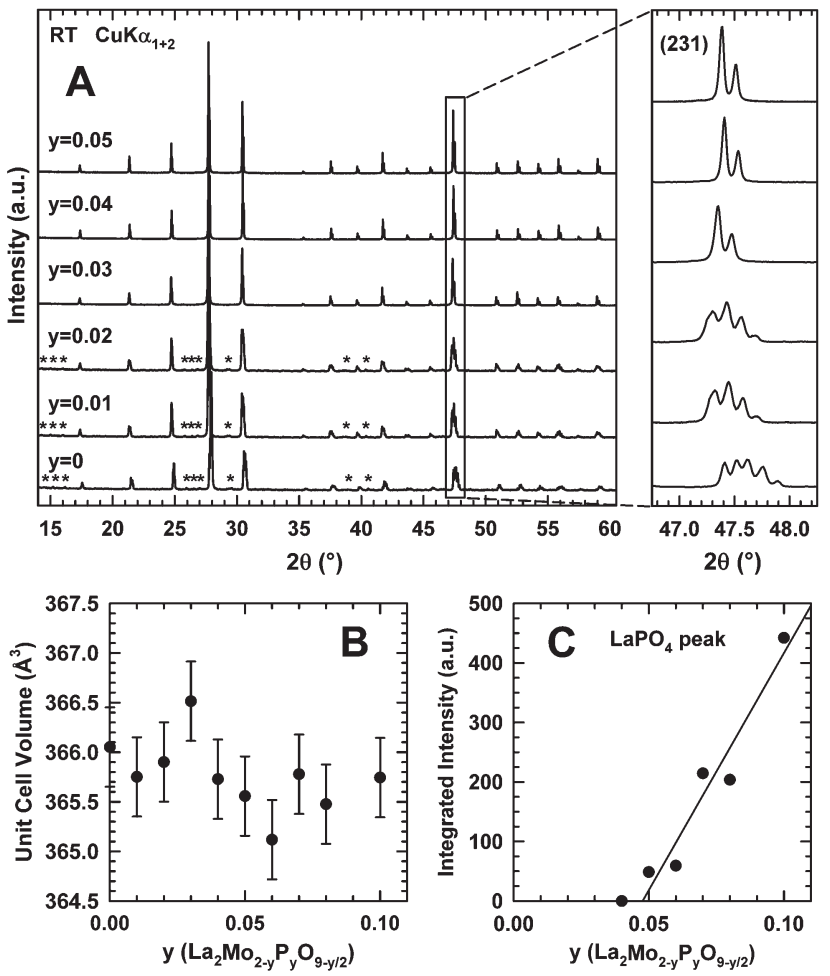

Fig. 1 (A) X-ray diffraction patterns of the $\mathrm{La}_{2} \mathrm{Mo}_{2-y} \mathrm{P}_{y} \mathrm{O}_{9-y / 2}$ series. Stars indicate superstructure peaks of the monoclinic cell. The excerpt at $46.5-48.5^{\circ}$ shows a phase transition from a monoclinic $(\alpha)$ phase to a cubic $(\beta)$ phase. (B) Unit cell volume as a function of $\mathrm{P}$ concentration. (C) Intensity of the (120) diffraction peak of $\mathrm{LaPO}_{4}\left(2 \theta=28.6^{\circ}\right.$, not visible in Fig. 1A) as a function of $\mathrm{P}$ concentration. The line corresponds to a linear regression fit of the data points representative of compositions where a impurity peak is observed $(y \geq 0.05)$.

the ideal case, a constant volume. Fig. 1B shows an overall decrease of the volume with $y$, but this decrease is hardly more distinct than the experimental error of each point. The limiting factor for the precision appears to be the mechanics of the diffractometer.

Therefore the major (120) peak of $\mathrm{LaPO}_{4}$ at 28.64 (PDF no. 01-084-0600) $)^{33}$ was recorded with high accuracy during very long scans (150 min scans, [27.2-29.2 ${ }^{\circ}$ range, $0.0084^{\circ}$ step). These extended scans revealed that the $y=0.05$ composition also contains a small amount of $\mathrm{LaPO}_{4}$ precipitate while for the $y=0.04$ composition no signal was detected. Fig. 1C displays the evolution of the $\mathrm{LaPO}_{4}$ amount as a function of $y$, the straight line corresponding to a linear regression of the points for which a non-zero intensity value was measured. It shows that the solid solution limit is just below $y=0.05$.

\section{${ }^{139}$ La NMR}

${ }^{139} \mathrm{La}(I=7 / 2)$ exhibits a sizeable quadrupolar coupling constant which usually leads to extremely broad signals that exceed by far the band width of the excitation pulses and the reception circuit. Spectra of $\mathrm{La}_{2} \mathrm{Mo}_{2-y} \mathrm{P}_{y} \mathrm{O}_{9-y / 2}$ with $y=0.00-0.06$ (in increments of 0.01$), 0.08$, and 0.10 were therefore recorded by sweeping the carrier frequency stepwise through the whole frequency range. Each sampled point displays the intensity at the carrier frequency

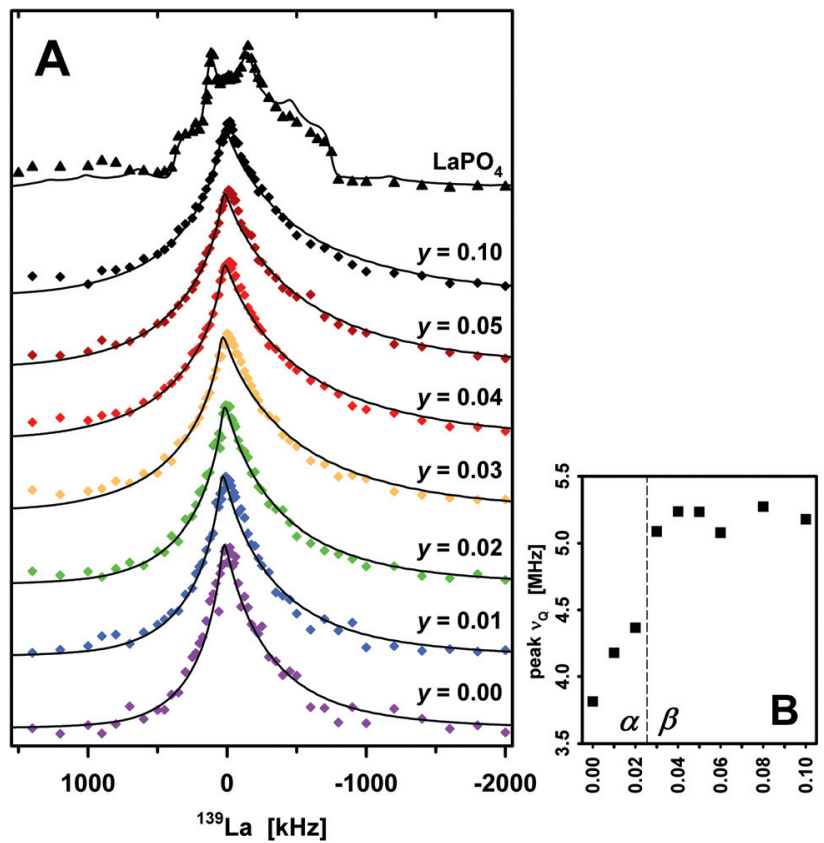

Fig. 2 (A) ${ }^{139} \mathrm{La}$ NMR spectra of $\mathrm{La}_{2} \mathrm{Mo}_{2-y} \mathrm{P}_{y} \mathrm{O}_{9-y / 2}$ for $y=0.00$ to 0.05 and 0.10 and the central transition of $\mathrm{LaPO}_{4}$. Black line: fitted model spectra. (B) Peak quadrupolar coupling constant as a function of the phosphorus content.

of the respective spectrum. In order to enhance the sensitivity, the time signal was sampled by means of the QCPMG scheme. ${ }^{24}$ Fig. 2A shows ${ }^{139} \mathrm{La}$ spectra of $\mathrm{La}_{2} \mathrm{Mo}_{2-y} \mathrm{P}_{y} \mathrm{O}_{9-y / 2}$ with $y=$ $0.00-0.05$ and 0.10 , and of the monazite $\mathrm{LaPO}_{4}$ for comparison. The monazite spectrum shows a broad central transition with distinct features of the second-order term of the quadrupolar coupling. They can be described by the corresponding standard model function with an enormous coupling constant $v_{\mathrm{Q}}=$ $3.4 \mathrm{MHz}$, an asymmetry parameter $\eta=0.68$ and a Gaussian line broadening of $64 \mathrm{kHz}$. The signals of $\mathrm{La}_{2} \mathrm{Mo}_{2-y} \mathrm{P}_{y} \mathrm{O}_{9-y / 2}$ are featureless, asymmetric, and have an unusual sharp summit. A tentative modeling with a crystal powder quadrupolar coupling model signal as for $\mathrm{LaPO}_{4}$ is not completely poor, but does not match these particular properties, and moreover yields implausible results as a quadrupolar coupling of only $v_{\mathrm{Q}}=0.4 \mathrm{MHz}$ for the parent compound, almost ten times smaller than that of $\mathrm{LaPO}_{4}$ and also much smaller than other ${ }^{139} \mathrm{La}$ coupling constants that can be found in the literature (see, for example, ref. 34). The coordination environment of $\mathrm{La}$ in $\mathrm{LAMOX}, 3,4$ however, does not make it likely that its electric field gradient has high cubic symmetry. As the line shape is rather untypical for polycrystalline materials, we try to model the spectra with a Czjzek distribution ${ }^{26}$ of the quadrupolar coupling, which is normally used for amorphous structures such as glasses. We focus here on the region dominated by the central transition. The best fits are achieved with a critical exponent $d=3$, reflecting that the distribution of the quadrupolar coupling obviously is not isotropic. The quadrupolar coupling constants at the peak of the distribution (Fig. 2B) are of the same order of magnitude as that of $\mathrm{LaPO}_{4}$. The model function represents quite well the special characteristics of the LAMOX ${ }^{139}$ La signal. The environments of the 48 crystallographically different La sites with their different 
(6 to 12) numbers of oxygen neighbours are thus so different that they lead to a broad distribution of the electric field gradient (EFG), for which a conventional crystal model (which would assume no distribution) is not appropriate. The fit however improved when broadening the lines with a broad chemical shift distribution: a width of $30 \mathrm{kHz}$, or a distribution of $\pm 350 \mathrm{ppm}$ around an average chemical shift of about +700 ppm, was appropriate for all $\mathrm{La}_{2} \mathrm{Mo}_{2-y} \mathrm{P}_{y} \mathrm{O}_{9-y / 2}$ spectra. This width appears relatively large and apparently compensates for the residual imperfection of the model.

Upon substitution of Mo with $\mathrm{P}$, the peak quadrupolar coupling initially increases with $y$. There is a significant jump from $y$ $=0.02$ to 0.03 , the point of phase transition from a monoclinic to cubic phase. The general shape however is preserved. Afterwards the peak coupling constant does not change any more (within the range of experimental uncertainty). The increase of

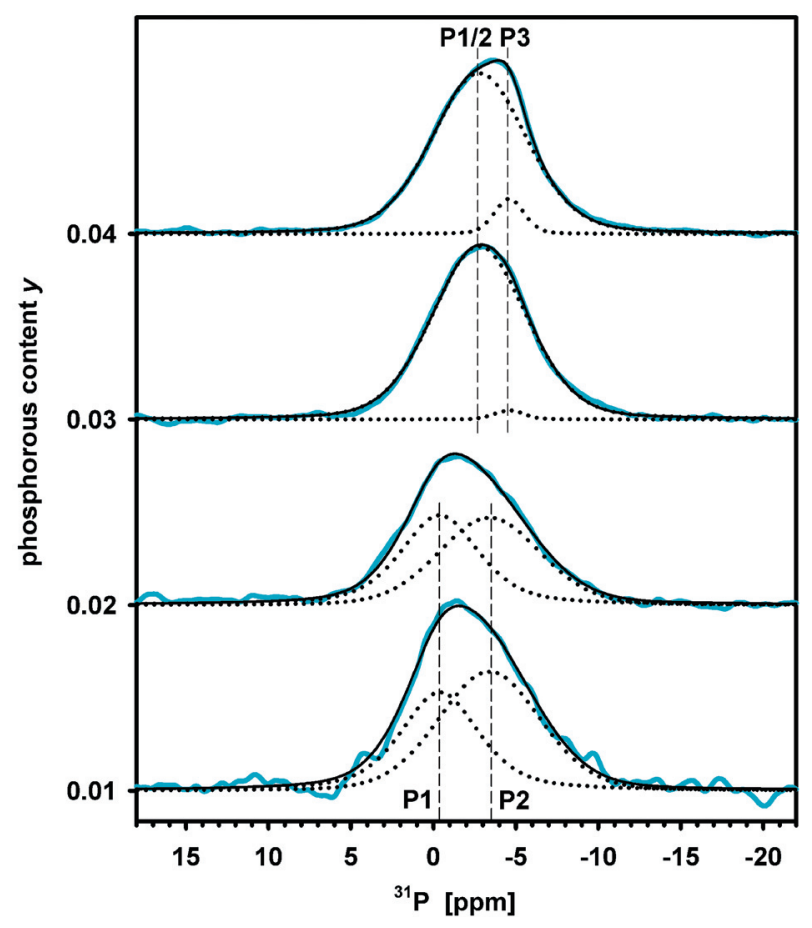

Fig. $3{ }^{31} \mathrm{P}$ NMR spectra of $\mathrm{La}_{2} \mathrm{Mo}_{2-y} \mathrm{P}_{y} \mathrm{O}_{9-y / 2}$ with $y=0.01$ to 0.04 , normalized to the $\mathrm{P}$ content (blue line). Deconvolution by joint fits of the two groups $y=0.01-0.02$ and $0.03-0.04$, respectively, with two signals each: black dotted lines: separate partial model signals, black solid lines: sum. the peak coupling constant with the phase transition is on the first glance counterintuitive, as one would expect that the quadrupolar coupling decreases with increasing crystal symmetry (compare for example $\mathrm{ZrO}_{2}$ in ref. 35), but the local symmetry is not necessarily correlated. The preservation of the amorphouslike shape of the signal rather shows that a local disorder remains and that the crystal structure represents, as suggested by Evans et al. for the high temperature $\beta$ phase of the parent compound, a dynamic average. ${ }^{4}$ The increase in the peak quadrupolar coupling will then be due to a higher average local symmetry distortion around the La nuclei, as it can be due to the higher number of possibilities to create an asymmetric La environment by oxygen vacancies of the $\mathrm{O} 2$ and $\mathrm{O} 3$ site.

\section{Quantitative ${ }^{31}$ P NMR}

Each ${ }^{31} \mathrm{P}$ single pulse spectrum of the series $y=0.01$ to 0.04 shows only one asymmetric peak, which has a maximum intensity that shifts with $y$ from about -1 to -4.5 ppm (Fig. 3). For deconvolution, the spectra were normalized to the $\mathrm{P}$ concentration (weighted by $1 / y$ ) in order to avoid an under-representation of the lower $y$ spectra in the error sum. One spectrum alone would not allow a detailed deconvolution, however we make use of the surplus of information given by the series of spectra that should have most parameters in common. We therefore perform in a first approach a curve fitting for all spectra together, assuming that peak positions and shapes are the same and that only the intensities of the peaks vary (Table 1, Fig. 4). These constraints reduce the number of parameters for a three site Lorentzian/Gaussian model from 48 ( 4 spectra $\times 3$ peaks $\times 4$ parameters, i.e., position, width, Gaussian percentage, intensity)

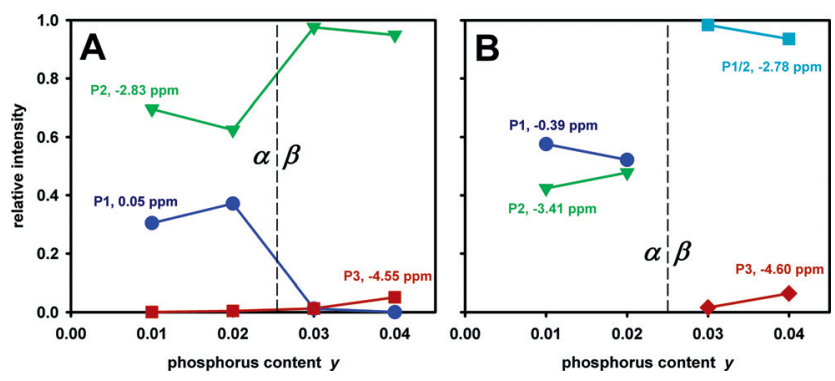

Fig. 4 Normalized populations of the three phosphorus sites as a function of the phosphorus content $y$. (A) Joint fit of all four ${ }^{31} \mathrm{P}$ spectra with a three-site model. (B) Joint fits of the two groups $y=0.01-0.02$ and $y=$ 0.03-0.04, respectively, with a two site model each.

Table 1 Common curve fit parameters in two different joint fit approaches. The intensities are shown in Fig. 4. The first approach is a three site model for all four spectra together, the second joins the spectra in the $\alpha$ and in the $\beta$ phase, respectively, in a two-site model. Errors are estimated to be in the order of $0.1 \mathrm{ppm}$ for chemical shifts and line widths, 20 percent points for the Gaussian fraction, and $10 \%$ for the intensities

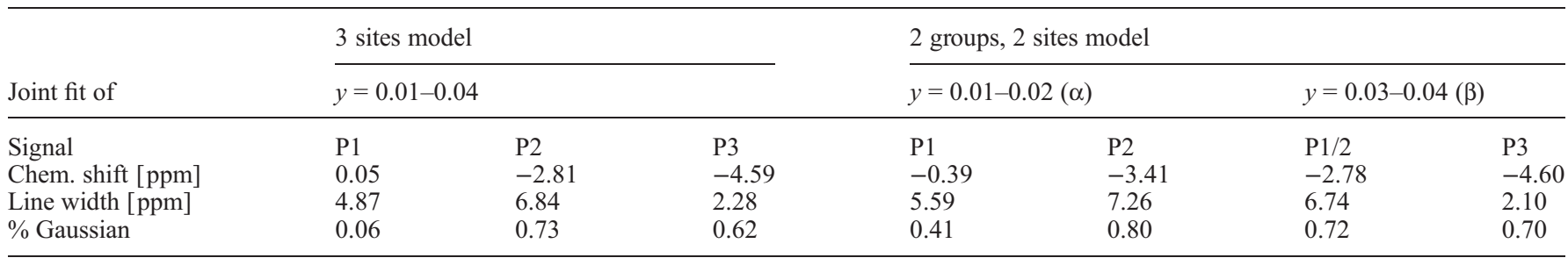


to 21 ( 3 peaks $\times 3$ common parameters, i.e. position, width, Gaussian percentage, plus the intensities of 3 peaks $\times 4$ spectra).

These constraints not only ensure a consistent result, but also allow and even require the refinement by proceeding from a twoto a three-site model: a two site model with 16 parameters yields, as may be expected by inspection, an upfield site whose population is increasing with $y$ and a downfield site decreasing with $y$ (Fig. 3). Each spectrum alone could be well described by a two-site model, but the constraints in a joint fit lead to an inappropriate model function for either low or high $\mathrm{P}$ concentration. Changing from a two-site to a three-site model leads to satisfactory fits for all spectra, and the error sum improves by $22 \%$.

Fig. 4A shows the three relative peak intensities as a function of $y$, representing three populations. At low $\mathrm{P}$ concentrations, the spectra can be described by two peaks at $0.05 \mathrm{ppm}$ (signal P1) and $-2.83 \mathrm{ppm}$ (signal $\mathrm{P} 2$ ). There is a discontinuity between $y=$ 0.02 and $y=0.03$; P1, contributing about $35 \%$ at low $\mathrm{P}$ concentration, vanishes beyond this point. This transition in the peak intensities coincides with the observed structural phase transition from the $\alpha$ to the $\beta$ phase between $y=0.02$ and 0.03 . Under this observation the fitting approach is not fully appropriate, as we give the constraint of same peak positions and shapes for the $\alpha$ and the $\beta$ phase. The observation that one site is vanishing while the other takes all the population may be induced by the above fitting approach. As a consequence, we adjust the fitting model by separating the four spectra into two groups, below $(y=$ $0.01-0.02)$ and above the phase transition $(y=0.03-0.04)$. As in each spectrum in the joint three-site approach only one or two of the three sites had a significant population, we reduce the number of sites to two in the separated approach (right column in Table 1). The error sum improves slightly by $5 \%$, with 20 rather than 21 parameters. Signals $\mathrm{P} 1$ and P2 now unify to a common peak $\mathrm{P} 1 / 2$ in the second group of spectra (Fig. 4B), representing a more homogeneous group of sites. However, at the same time a signal at $-4.60 \mathrm{ppm}$ (signal P3) arises, increasing with the $\mathrm{P}$ concentration.

We have acquired ${ }^{31} \mathrm{P}$ NMR spectra of several reference compounds, the most relevant being the impurity $\mathrm{LaPO}_{4}$ found in the $\mathrm{La}_{2} \mathrm{Mo}_{2-y} \mathrm{P}_{y} \mathrm{O}_{9-y / 2}$ series for $y=0.05$ or higher. $\mathrm{LaPO}_{4}$ gives a line at $-4.56 \mathrm{ppm}$, similar to signal P3, indicating that P3 not only has 4-fold coordination but also a very similar or identical chemical environment as $\mathrm{LaPO}_{4}$. In fact, at a concentration $y=$ 0.10 this signal becomes the dominating contribution. The straightforward interpretation is thus precipitation of $\mathrm{LaPO}_{4}$ from $y=0.04$, which is however in contradiction to the outcome of the X-ray diffraction study.

In order to test whether the site heterogeneity is due to the presence of hydroxide ions in the coordination sphere of the phosphorus ion in the LAMOX phase, spectra of $\mathrm{NH}_{4} \mathrm{H}_{2} \mathrm{PO}_{4}$ and $\left(\mathrm{NH}_{4}\right)_{2} \mathrm{HPO}_{4}$ were acquired, whose signals however did not show any similarity (0.88 and $1.24 \mathrm{ppm})$.

\section{Exchange experiments}

The ${ }^{31} \mathrm{P}$ nuclei in the compounds $\mathrm{La}_{2} \mathrm{Mo}_{2-y} \mathrm{P}_{y} \mathrm{O}_{9-y / 2}$ have long relaxation times $T_{1}$ in the order of some minutes, which are favourable for chemical exchange experiments to study the ionic motions in solids. When an $\mathrm{O}^{2-}$ ion moves to or away from a $\mathrm{P}$ site, the coordination of the respective $\mathrm{P}$ sites and the corresponding chemical shift should change. If this chemical exchange is faster than the NMR time scale, the signal frequencies are averaged. However, if it is slower, 2D exchange experiments can show correlations between $\mathrm{P}$ ions with different coordinations by cross peaks in the $2 \mathrm{D}$ spectrum. The exchange experiments were performed with the samples with $y=0.03$ and 0.04 with mixing times of $1 \mathrm{~ms}$ to $60 \mathrm{~s}$. However, all the experiments did not show any exchange cross peaks between P1/2 and $\mathrm{P} 3$, neither at room temperature nor at higher temperature $(350 \mathrm{~K})$.

\section{${ }^{31} \mathbf{P}-{ }^{31} \mathbf{P}$ coupling}

In order to understand the nature of P3 as well as the lack of exchange with $\mathrm{P} 1 / 2$, a $2 \mathrm{D}$ double-quantum-single-quantum (DQ-SQ) experiment has been conducted by means of POST-C $7^{28}$ recoupling for the composition $y=0.04$ (Fig. 5A). The spectrum shows one cross peak at $-4.6 \mathrm{ppm}$ SQ resonance (P3) correlated to its own DQ resonance position (about $-9.2 \mathrm{ppm}$ ). In contrast, cross peaks between P3 and P1/2 or P1/2 and itself cannot be observed. The existence of a cross-peak indicates that P3-type of P atoms are relatively close to each other, giving rise to dipolar connectivity. A P-P distance of about $4.5 \AA$, the average distance between two neighbouring $\mathrm{Mo} / \mathrm{P}$ sites, causes a dipolar coupling of about $200 \mathrm{~Hz}$, just sufficient for a transfer with an adequate recoupling sequence. P1/2 type $\mathrm{P}$ atoms however must be farther apart from each other and from P3 because the respective dipolar coupling is too weak to excite DQ coherences. The existence of cross peaks thus indicates a clustering of $\mathrm{P} 3$-type phosphorus on neighbouring $\mathrm{Mo} / \mathrm{P}$ sites.
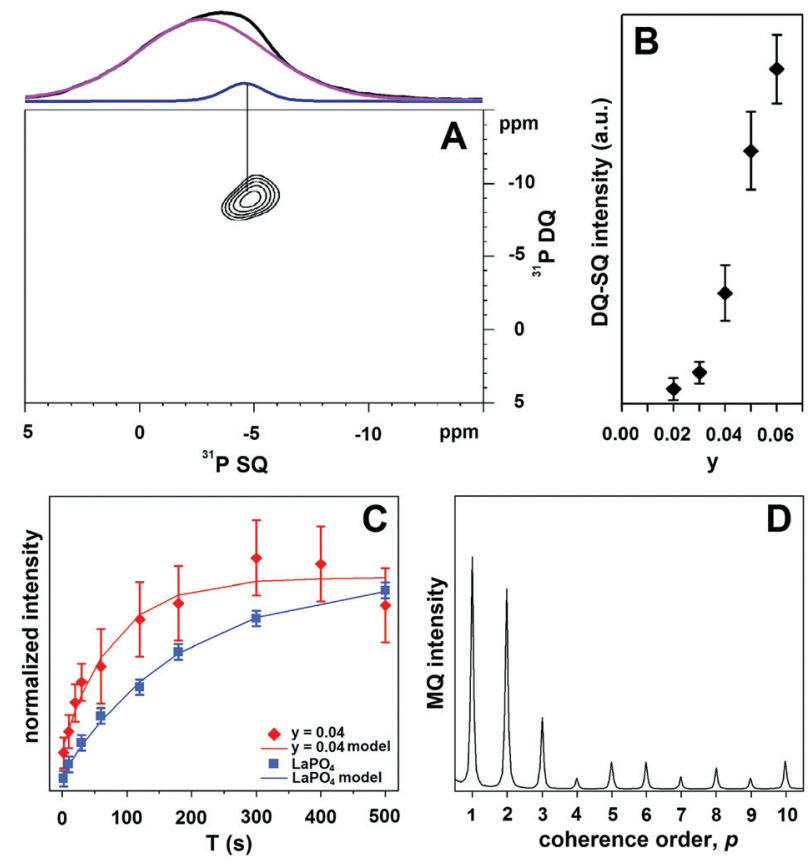

Fig. 5 (A) ${ }^{31} \mathrm{P}$ DQ-SQ MAS spectrum of $\mathrm{La}_{2} \mathrm{Mo}_{2-y} \mathrm{P}_{y} \mathrm{O}_{9-y / 2}$ with $y=$ 0.04 . On top $1 \mathrm{D}$ signal (black) with deconvolution (violet, blue) (B) Intensity of the DQ-SQ peak $(2 \mathrm{P} 3 \times \mathrm{P} 3)$ as a function of $y$. (C) Saturation recovery of the DQ-filtered $\mathrm{P} 3$ signal of $y=0.04$ and of $\mathrm{LaPO}_{4}$. Relaxation times, $y=0.04: T_{1}=78 \pm 20 \mathrm{~s}$; $\mathrm{LaPO}_{4}: T_{1}=186 \pm 10 \mathrm{~s}$. (D) Multiple-quantum excitation (spin counting) diagram. 
Fig. 5B shows the intensity of the cross peak as a function of $y$ (it should therefore be correlated to the behaviour of the P3 signal in Fig. 4B). After sufficiently long acquisition, a significant DQ-SQ signal was observed already at $y=0.03$, and for $y=0.02$ there appears to be a signal just above noise level. In order to resolve the apparent contradiction with the diffraction results and to study the P3 signal in more detail, we have measured the longitudinal relaxation times of the P3 signal in $y=0.04$ and of $\mathrm{LaPO}_{4}$. The separation from the other signals was achieved by a DQ-filter. As the saturation recovery curves in Fig. 5C show, the relaxation of the P3 signal in $y=0.04$ is significantly faster than that of $\mathrm{LaPO}_{4}$. The times are $T_{1}=78 \mathrm{~s}$ for $y=0.04$ and $186 \mathrm{~s}$ for $\mathrm{LaPO}_{4}$.

\section{Excitation of multiple quantum ${ }^{31} P$ coherences}

In principle, already in a homogeneous distribution of $\mathrm{P}$ there is a certain probability that two $\mathrm{P}$ atoms replace two Mo on neighbouring sites. If this had been the reason for the observed cross peaks, one should see DQ-SQ cross peaks also for the site P1/2. As a further test, we tried to excite higher order multiplequantum coherences. These spin counting experiments, usually applied for ${ }^{1} \mathrm{H}$ with their strong couplings, are not very sensitive for ${ }^{31} \mathrm{P}$. For $\mathrm{LaPO}_{4}$ up to 5 -quantum coherences could be excited (data not shown). Fig. 5D shows a multiple-quantum diagram of the P3 signal of the compound with $y=0.04$, acquired by means of BABA recoupling. ${ }^{29,30}$ Coherences of 1,2 and maximal 3 quanta could be excited with an intensity that is significantly above noise, while corresponding coherences for P1/2 were absent except for the single-quantum signal (not shown). Note that the zero-quantum peaks can contain baseline artefacts and are therefore omitted in the graph. The low statistical probability in connection with the low efficiency of 3-quantum excitation make it unlikely that the 3-quantum signal in Fig. 5D, about one third of the 2-quantum signal, stems from phosphorus atoms in a homogeneous distribution on Mo sites. It therefore confirms that there are clusters of at least three P3 phosphorus atoms on neighbouring Mo sites in the $y=0.04$ P-substituted LAMOX. Note that this does not mean by any means a limitation to three; this number is only the experimental limit of multiple-quantum excitation.

\section{Discussion}

The results of the different ${ }^{31} \mathrm{P}$ NMR experiments, in particular the multiple-quantum excitation of P3 and the lack of an exchange peak demand a principle distinction between the character of the P3 site on the one hand and the P1 and P2 sites on the other hand. The P1 and P2 phosphorus are distributed randomly over the Mo lattice positions. At higher $\mathrm{P}$ concentrations ( $\beta$ phase), they can be described by one model signal, while below the phase transition concentration ( $\alpha$ phase) two are necessary. Line widths and spectral overlap in combination with signal-to-noise ratio, however, do not allow an interpretation in terms of exactly two distinct phosphorus sites. In contrast, a model of two peaks is sufficient to describe a potential multitude of sites, for which more detailed information is not obtainable. In fact, the LAMOX $\alpha$ phase contains 48 sites divided into three groups. The phase transition from $\alpha$ to $\beta$ phase is reflected in both ${ }^{139} \mathrm{La}$ and ${ }^{31} \mathrm{P}$ NMR spectra. Evans et al. have proposed the hypothesis that the $\beta$ phase of the parent compound is a dynamic average of the $\alpha$ phase. ${ }^{4}$ This would match with the transition to only one average ${ }^{31} \mathrm{P} \mathrm{NMR}$ site at the $y=0.03 \mathrm{P}$-substituted $\beta$ phase. Earlier crystal structure analysis ${ }^{3,4}$ of the $\mathrm{La}_{2} \mathrm{Mo}_{2} \mathrm{O}_{9}$ parent compound in its $\beta$ phase showed that two of the three oxygen sites (O2 and $\mathrm{O} 3$ ) are partially occupied and one (O1) is fully occupied. In the $\alpha-\mathrm{La}_{2} \mathrm{Mo}_{2} \mathrm{O}_{9}$ phase, the Mo atoms were found in three different basic coordination types of 4, 5 and 6. Under normal conditions, phosphorus in phosphate compounds can however only be surrounded by four oxygens due to its small size. On the other hand one can argue that in LAMOX compounds the structure and distances are dominated by the Mo properties due to the low concentration of the substituting element, and that therefore $\mathrm{P}$ could take the coordination of the atom it replaces. The phosphorus chemical shifts in P-substituted LAMOX only show relatively little dispersion when compared to the overall chemical shift range of ${ }^{31} \mathrm{P}$. This rather indicates that phosphorus remains in its preferred 4-fold coordination at all concentrations. For comparison, we refer to the behaviour of yttrium in YSZ, where ${ }^{89}$ Y NMR shows a shift from 8- to 7- and 6-fold coordination, significant however only beyond much higher concentrations of $20 \%$ (7-fold) and $40 \%$ (6-fold) $\mathrm{Y}_{2} \mathrm{O}_{3}$ in zirconia. $^{36}$

The possibility to excite multiple-quantum coherences of P3 and the lack of exchange peaks between $\mathrm{P} 3$ and $\mathrm{P} 1 / 2$ indicate the formation of clusters in which Mo is substituted by P. The P3 signal is similar to that of $\mathrm{LaPO}_{4}$. The NMR data alone could thus be interpreted in terms of a precipitation of $\mathrm{LaPO}_{4}$ grains. However, saturation observable by X-ray diffraction begins only at about $y=0.048$ (Fig. 1C) as compared to the observation of a DQ-SQ signal already at $y=0.03$ or even 0.02 (Fig. 5B). The different longitudinal relaxation behaviour (Fig. 5C) indicates if one presumes a similar chemical environment - a different mobility of the phosphorus in $\mathrm{P} 3$ compared to $\mathrm{LaPO}_{4}$. It makes it unlikely that $\mathrm{P} 3$ stems from real $\mathrm{LaPO}_{4}$ grains. A solution to this apparent contradiction could be a low dimensional (non-3D) clustering of $\mathrm{P}$ atoms on Mo sites. In the first article on LAMOX oxide ion conductors, ${ }^{1}$ a comparison was made between the cationic arrangement of the $\beta-\mathrm{La}_{2} \mathrm{Mo}_{2} \mathrm{O}_{9}$-type structure and that of the $\mathrm{LaPO}_{4}$ monazite structure: each can be considered as the periodic micro-twinning of the other, through cationic planes parallel to a LAMOX crystal cell face. One can therefore consider that, if $\mathrm{P}$ cations tend to cluster in a LAMOX-type structure, they will preferentially occupy such a plane and induce a twinning corresponding to a $\mathrm{LaPO}_{4}$-type slab between two LAMOX type grains (see Fig. 6). This type of clustering has a 2D dimensionality. Above such a substitution rate of $2 \mathrm{~mol} \%$ (i.e. $1.9 \mathrm{~mol} \% \mathrm{P}$ randomly distributed in LAMOX grains and $0.1 \mathrm{~mol} \% \mathrm{P}$ clustered in twinning planes), the clustering loses its $2 \mathrm{D}$ character, which results in $3 \mathrm{D} \mathrm{LaPO}_{4}$ crystallites as detected by X-ray diffraction.

TEM images of the $y=0.05$ sample, recorded on a $200 \mathrm{kV}$ side-entry JEOL 2010 electron microscope, show that the grains are formed of domains with size ranging from 5 to $100 \mathrm{~nm}$. The domains, well crystallized, are slightly disoriented with respect to each other. The reason for this domain formation, not observed in any other cubic substituted LAMOX phase, might 


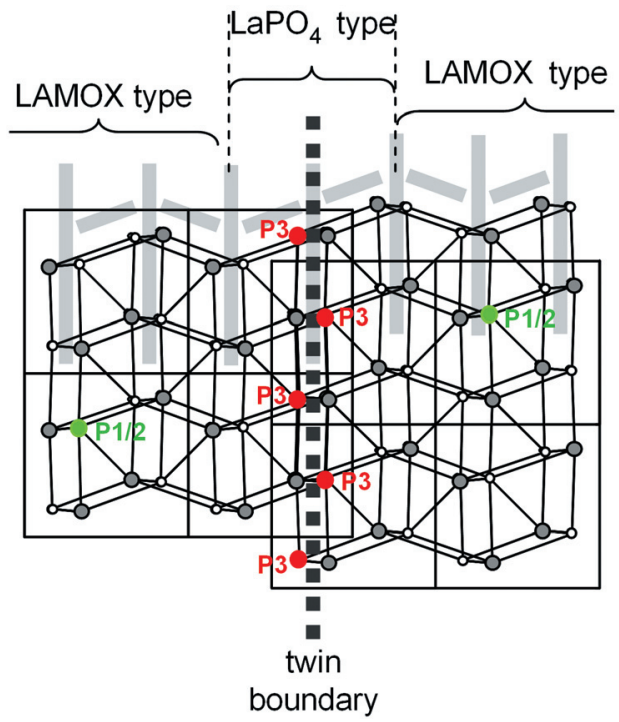

- $\mathrm{La}^{3+}$ in LAMOX or $\mathrm{LaPO}_{4}$

- $\mathrm{P}^{5+}$ in $\operatorname{LAMOX}(\mathrm{P} 1 / 2)$

- $\mathrm{Mo}^{6+}$ in LAMOX

- $\mathrm{P}^{5+}$ in $\mathrm{LaPO}_{4}(\mathrm{P} 3)$

Fig. 6 Hypothetical model of $\mathrm{La}_{2} \mathrm{Mo}_{2} \mathrm{O}_{9} / \mathrm{LaPO}_{4} / \mathrm{La}_{2} \mathrm{Mo}_{2} \mathrm{O}_{9}$ twinning consistent with both NMR and X-ray diffraction results. Compare with the LAMOX and $\mathrm{LaPO}_{4}$ structures in ref. 1. Only the cations are shown, oxygens are omitted in order to simplify the figure. The twinning between two $\mathrm{La}_{2} \mathrm{Mo}_{2} \mathrm{O}_{9}$-type domains creates a $2 \mathrm{D} \mathrm{LaPO}_{4}$-type slab.

be related to distortions caused by the formation of $\mathrm{LaPO}_{4}$-type planes of a limited extension.

\section{Conclusions}

LAMOX with molybdenum substitution by phosphorus is a representative of cationic substitution systems where saturation is already reached at low concentrations. It is a favorable example for NMR studies of the structural differences induced by a low amount of substituent because of the relatively high sensitivity of ${ }^{31} \mathrm{P}$ in NMR and the high sensitivity of the large quadrupolar coupling of ${ }^{139} \mathrm{La}$ towards changes in the environment. Substitutions between 0.5 and $5 \%$ are a challenge not only for NMR; also X-ray diffraction is in some respects at the limit of precision, in particular with respect to the small changes of the unit cell volume parameter. Our study shows how observations with one technique are detectable in the other one: while the phase transition from $\alpha$ to $\beta$ crystallographic phase is of course very easily detectable and identifiable by X-ray diffraction, it is a secondary effect - but visible - in ${ }^{31} \mathrm{P}$ NMR, and it is reflected as a sudden increase in the mean quadrupolar coupling of ${ }^{139} \mathrm{La}$. The multitude of crystallographic La sites as well as the statistics in occupation of the oxygen sites leads to ${ }^{139} \mathrm{La}$ signals that rather resemble those of amorphous rather than crystalline phases; the quadrupolar couplings apparently follow a relatively broad distribution. Well comparable - and controversial - is the determination of the saturation limit of phosphorus: while diffraction does not show a typical signal of $\mathrm{LaPO}_{4}$ precipitates below $y=$ $0.05,{ }^{31} \mathrm{P} \mathrm{NMR}$ shows a $\mathrm{LaPO}_{4}$-like signal and moreover couplings which identify neighbouring $\mathrm{P}$ atoms already at $y=0.03$.
${ }^{31} \mathrm{P}$ relaxation however indicates that the environment around the respective $\mathrm{P}$ atom is not completely identical with $\mathrm{LaPO}_{4}$. We try to explain the apparent contradiction by a hypothetical model that contains two-dimensional $\mathrm{LaPO}_{4}$ areas as interfaces of crystal twinning, representing the beginning of precipitation which only at higher concentrations becomes three-dimensional and thus detectable by diffraction.

\section{Acknowledgements}

The authors would like to thank Dr Monique Body and Prof. Alain Bulou for valuable discussions and Dr Stéphanie Kodjikian for collecting TEM images. The financial support of the Region Pays de la Loire is gratefully acknowledged (Convention No. 2007-11860).

\section{Notes and references}

1 P. Lacorre, F. Goutenoire, O. Bohnke, R. Retoux and Y. Laligant, Nature, 2000, 404, 856.

2 F. Goutenoire, O. Isnard, E. Suard, O. Bohnke, Y. Laligant, R. Retoux and P. Lacorre, J. Mater. Chem., 2001, 11, 119.

3 F. Goutenoire, O. Isnard, R. Retoux and P. Lacorre, Chem. Mater, 2000, 12, 2575.

4 I. R. Evans, J. A. K. Howard and J. S. O. Evans, Chem. Mater, 2005, 17, 4074.

5 P. Lacorre, Solid State Sci., 2000, 2, 755.

6 S. Georges, F. Goutenoire, O. Bohnke, M. C. Steil, S. J. Skinner, H.D. Wiemhöfer and P. Lacorre, J. New. Mat. Electrochem. Systems, 2004, 7,51 .

7 L. Malavasi, H. J. Kim, S. J. L. Billinge, T. Proffen, C. Tealdi and G. Flor, J. Am. Chem. Soc., 2007, 129, 6903.

8 C. Tealdi, G. Chiodelli, L. Malavasi and G. Flor, J. Mater. Chem., 2004, 14, 3553 .

9 X. P. Wang, Z. J. Cheng and Q. F. Fang, Solid State Ionics, 2005, 176, 761.

10 D. Marrero-Lopez, D. Perez-Coll, J. C. Ruiz-Morales, J. CanalesVazquez, M. C. Martin-Sedeno and P. Nunez, Electrochim. Acta, 2007, 52, 5219; A. Selmi, C. Galven, G. Corbel and P. Lacorre, Dalton Trans., 2010, 39, 93.

11 X. P. Wang, Q. F. Fang, Z. S. Li, G. G. Zhang and Z. G. Yi, Appl. Phys. Lett., 2002, 81, 3434.

12 A. Subramania, T. Saradha and S. Muzhumathi, J. Power Sources, 2007, 167,319

13 S. Georges, F. Goutenoire, F. Altorfer, D. Sheptyakov, F. Fauth, E. Suard and P. Lacorre, Solid State Ionics, 2003, 161, 231.

14 D. Marrero-Lopez, J. Canales-Vazquez, W. Z. Zhou, J. T. S. Irvine and P. Nunez, J. Solid State Chem., 2006, 179, 278; G. Corbel, P. Durand and P. Lacorre, J. Solid State Chem., 2009, 182, 1009.

15 D. Marrero-Lopez, P. Nunez, M. Abril, V. Lavin, U. R. RodriguezMendoza and V. D. Rodriguez, J. Non-Cryst. Solids, 2004, 345-346, 377; G. Corbel, E. Chevereau, S. Kodjikian and P. Lacorre, Inorg. Chem., 2007, 46, 6395.

16 V. I. Voronkova, E. P. Kharitonova and A. E. Krasil'nikova, Crystallogr. Rep., 2010, 55, 276.

17 S. Basu, P. S. Devi and H. S. Maiti, J. Electrochem. Soc., 2005, 152, A2143.

18 Z. S. Khadasheva, N. U. Venskovskii, M. G. Safronenko, A. V. Mosunov, E. D. Politova and S. Y. Stefanovich, Inorg. Mater., 2002, 38, 1168.

19 G. Corbel, Y. Laligant, F. Goutenoire, E. Suard and P. Lacorre, Chem. Mater, 2005, 17, 4678 .

20 J. A. Collado, M. A. G. Aranda, A. Cabeza, P. Olivera-Pastor and S. Bruque, J. Solid State Chem., 2002, 167, 80.

21 A. Arulraj, F. Goutenoire, M. Tabellout, O. Bohnke and P. Lacorre, Chem. Mater, 2002, 14, 2492.

22 J. Rodriguez-Carvajal, Phys. B, 1993, 192, 55.

23 A. Le Bail, H. Duroy and J. L. Fourquet, Mater. Res. Bull., 1988, 23, 447. 
24 H. Y. Carr and E. M. Purcell, Phys. Rev., 1954, 94, 630; S. Meiboom and D. Gill, Rev. Sci. Instrum., 1958, 29, 688; F. H. Larsen, H. J. Jakobsen, P. D. Ellis and N. C. Nielsen, J. Phys. Chem. A, 1997, 101, 8597.

25 D. Massiot, F. Fayon, M. Capron, I. King, S. Le Calvé, B. Alonso, J-O. Durand, B. Bujoli, Z. Gan and G. Hoatson, Magn. Reson. Chem., $2002, \mathbf{4 0}, 70$.

26 G. Czjzek, J. Fink, F. Götz, H. Schmidt, J. M. D. Coey, J. P. Rebouillat and A. Liénard, Phys. Rev. B, 1981, 23, 2513.

27 D. R. Neuville, L. Cormier and D. Massiot, Geochim. Cosmochim. Acta, 2004, 68, 5071.

28 M. Hohwy, H. J. Jakobsen, M. Edén, M. H. Levitt and N. C. Nielsen, J. Chem. Phys., 1998, 108, 2686.

29 M. Munowitz and A. Pines, Science, 1986, 233, 525; C. E. Hughes, Prog. Nucl. Magn. Reson. Spectrosc., 2004, 45, 301.
30 M. Feike, D. E. Demco, R. Graf, J. Gottwald, S. Hafner and H. W. Spiess, J. Magn. Reson., Ser. A, 1996, 122, 214.

31 D. N. Shykind, J. Baum, S. B. Liu and A. Pines, J. Magn. Reson., 1988, 76, 149.

32 R. D. Shannon, Acta Crystallogr., Sect. A: Cryst. Phys., Diffr., Theor. Gen. Crystallogr., 1976, 32, 751.

33 PDF-4 + 2009; The International Centre for Diffraction Data (ICDD), 12 Campus Boulevard, Newtown Square, Pennsylvania 190733273 , USA.

34 T. J. Bastow, Solid State Nucl. Magn. Reson., 1994, 3, 17.

35 M. E. Smith and E. R. H. van Eck, Prog. Nucl. Magn. Reson. Spectrosc., 1999, 34, 159.

36 K. Kawara, H. Maekawa, T. Nemoto and T. Yamamura, Solid State Ionics, 2006, 177, 1687. 